\title{
Plasmon-Enhanced Four-Wave Mixing Imaging for Microdroplet-based Single-cell Analysis
}

Lili Conga ${ }^{\mathrm{a}}$ Yijia Geng ${ }^{\mathrm{a}}$, Yu Tian ${ }^{\mathrm{a}}$, Zepeng Huo ${ }^{\mathrm{a}}$, Dianshuai Huang ${ }^{\mathrm{b}}$, Chongyang Liang ${ }^{\mathrm{b}}$, Weiqing $\mathrm{Xu}^{\mathrm{a}}$, Yuling Wang ${ }^{\mathrm{c}}$, and Shuping $\mathrm{Xu}^{\mathrm{a}, \mathrm{c}, *}$

${ }^{\text {a }}$ State Key Laboratory of Supramolecular Structure and Materials, Institute of Theoretical Chemistry, College of Chemistry, Jilin University, Changchun 130012, China

${ }^{\mathrm{b}}$ Institute of Frontier Medical Science, Jilin University, Changchun 130021, China

${ }^{c}$ Department of Molecular Sciences, ARC Centre of Excellence for Nanoscale BioPhotonics (CNBP), Macquarie University, Sydney 2109, Australia

*Corresponding authors

Address: State Key Laboratory of Supramolecular Structure and Materials, Jilin University, 2699 Qianjin Ave. Changchun 130012, P. R. China

Email: xusp@jlu.edu.cn

Fax: 86-431-85193421 


\section{Table of contents}

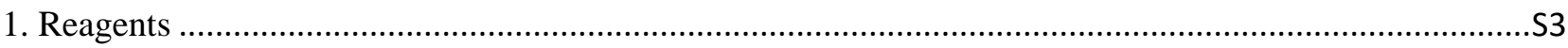

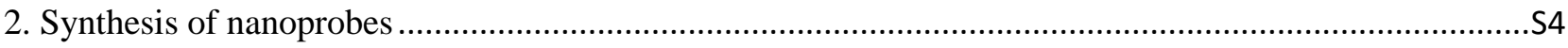

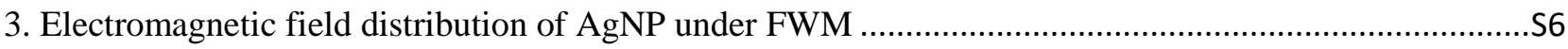

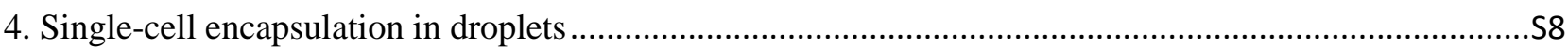

5. Dark-field imaging and bright-field imaging (particle-finder) .............................................................

6. Fluorescence imaging of EGFR on cell surface ...............................................................................

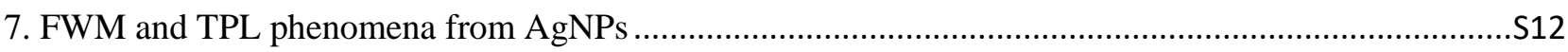

8. FWM imaging of single cell contained droplet array and automatic image processing by Image $\mathrm{J}$.........S14

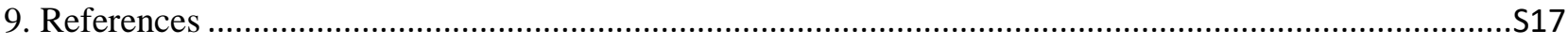




\section{Reagents}

Silver nitrate $\left(\mathrm{AgNO}_{3}>99.8 \%\right)$, trisodium citrate dehydrate $(99 \%)$ and Tween 20 were purchased from Shanghai Chemical Reagent Co., Ltd. Ethanolamine (97\%), 1-ethyl-3-(3-dimethylaminopropyl)carbodiimide hydrochloride (EDC, 99\%), N-hydroxysuccinimide (NHS, 98\%), 4-mercaptophenylboronic acid (MPBA) were bought from Aladdin Industrial Corporation. Poly(dimethyldiallylammonium chloride) (PDDA) was purchased from Sigma-Aldrich. EGFR monoclonal antibody (111.6) was acquired from Thermo Fisher Scientific Co., Ltd. The Alexa Fluor ${ }^{\circledR} 488$ donkey anti-mouse $\operatorname{IgG}(\mathrm{H}+\mathrm{L})$ antibody was bought from Life Technologies (USA). Iscove's Modified Dulbecco's Medium (IMDM, Gibcos) was purchased from Life Technologies Corporation. Phosphate-buffered saline (PBS) solution (0.1 M, pH=7.4) was prepared for cell experiments. The deionized water used was purified by the Millipore system.

SU8-2075 was purchased from Wenhao Co., Ltd. Developing agent solution was obtained from Shenzhen Donggangtian chemical Co. Ltd (Shenzhen, China). Silicone elastomer and silicone elastomer curing agent were purchased from Dow Corning (Midland, Michigan, USA). Aquapel solution was bought from PPG Industries (Pittsburgh, PA). Perfluoropolyether-polyethylene glycol (PFPE-PEG) block copolymer surfactant was obtained from Ranbiotech. Optiprep medium was purchased from Sigma-Aldrich.

HepG2 (human liver cancer cell carcinoma), LO2 (human normal liver cell line), HeLa (human cervical cancer cells) cells were both bought from Shanghai ATCC cell bank which has been issued with the permission of the Human Research Ethics Committee of the country for manipulations of human's cells. 


\section{Synthesis of nanoprobes}

Silver nanoparticles (AgNPs) were synthesized according to Lee's method. ${ }^{[1]}$ The Ag@MPBA nanoprobe was synthesized by coating MPBA on the surface of AgNPs, which has been stated in our previous publication. ${ }^{[2]}$ Mainly, $3.4 \mu \mathrm{L}$ of the MPBA ethanol solution $(1.0 \mathrm{mM})$ was added into $10.0 \mathrm{~mL}$ of AgNPs $\left(1.808 \times 10^{-11} \mathrm{M}\right)$, in which the coverage of MPBA on the surface of AgNPs is about 0.6 according to the $50 \mathrm{~nm}$-sizedAgNPs. After 15 min of vigorous stirring, the mixture was left for $12 \mathrm{~h}$ aging in dark. In order to remove the excess reactants from the sample, the solution was centrifuged at $5800 \mathrm{rpm}$ for $7 \mathrm{~min}$. Then the supernatant was removed, and the precipitates were re-dispersed in deionized water.
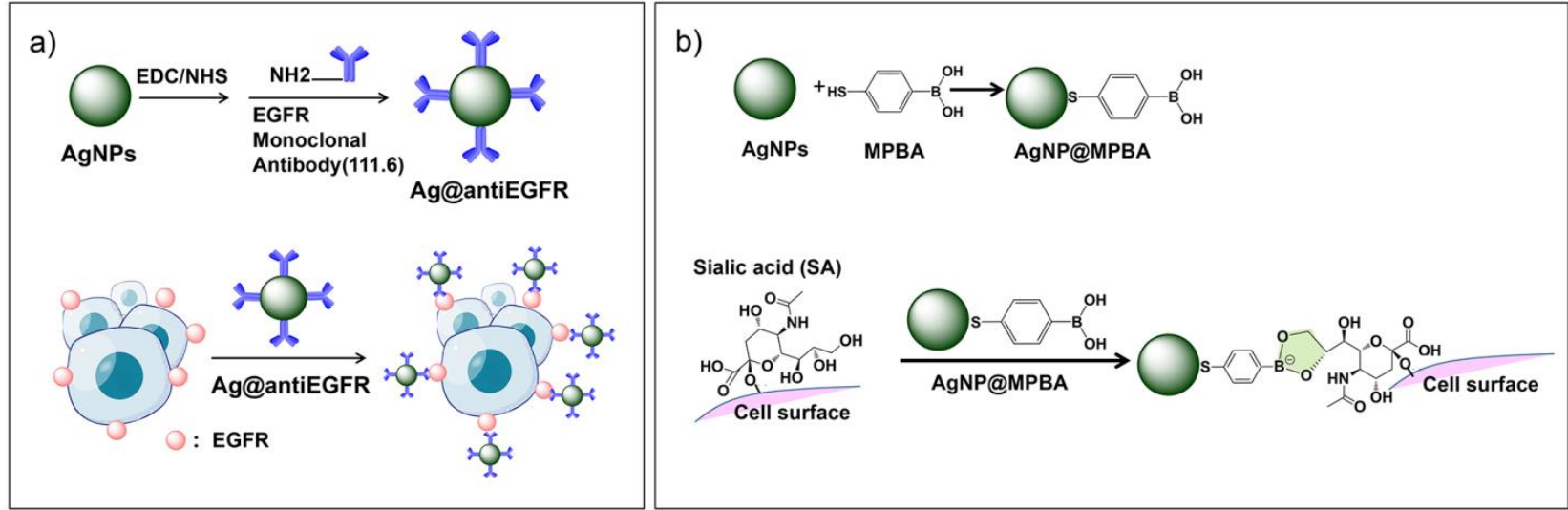

Scheme S1 Two surface reactions of two nanoprobes above the cell membrane.

The Ag@anti-EGFR nanoprobe was fabricated by the surface modification of the EGFR monoclonal antibody (111.6, anti-EGFR) on the surface of AgNPs according to the literature ${ }^{[3]}$ Briefly, $10 \mu \mathrm{L}$ of Tween 20 was added to $1.0 \mathrm{~mL}$ of AgNPs $(0.2 \mathrm{nM})$ solution with rapid stirring for $30 \mathrm{~min}$ at room temperature to prevent the aggregation of AgNPs during surface modification. The carboxylate terminal groups on the surfaces of AgNPs were activated by adding $5.0 \mu \mathrm{L}$ of EDC and NHS (both $0.1 \mathrm{M}$ ), and then $4 \mu \mathrm{L}$ of $0.05 \mathrm{mg} / \mathrm{mL}$ anti-EGFR were mixed the above AgNPs solution for $4 \mathrm{~h}$. The unreacted molecules were first removed from the solutions by centrifugation at 6000 rpm for 7 min. Finally, unreacted sites on the surfaces of the AgNPs were deactivated by $2.0 \mu \mathrm{L}$ of ethanolamine (97\%) for $20 \mathrm{~min}$. Nonspecific binding chemicals and antibodies were washed with deionized water twice to eliminate any excess reagents, and AgNPs@anti-EGFR nanoprobes (60 pM) were dispersed in deionized water for experimental use. According to the literature, ${ }^{[3]}$ the loaded antibodies would be about $3 \sim 4$ per AgNP.

The morphology and size of AgNPs were characterized by JEM-2100F field-emission transmission electron 
microscopy (TEM, JEOL, Tokyo, Japan) and the result is shown in Figure S1a. It can be observed that the AgNPs are in various shapes and the semi-spherical shape is dominated. Since the preparation of nanoprobes experiences several times centrifugation, too small and too large sized AgNPs (rod type) will be partly removed. The plasmonic property of AgNPs, Ag@MPBA, and Ag@anti-EGFR were obtained by ultraviolet-visible (UV-vis, Ocean Optics, USB4000). It should be noted that their profiles are not exactly the same since AgNPs are from different trials, however, they won't affect the results of FWM imaging. The maximum absorption of Ag@MPBA red-shifts as about $4 \mathrm{~nm}$ (Figure S1b) and the conjugating monoclonal anti-EGFR antibody with AgNPs has a red-shift of about $6 \mathrm{~nm}$ relative to the bare AgNPs.
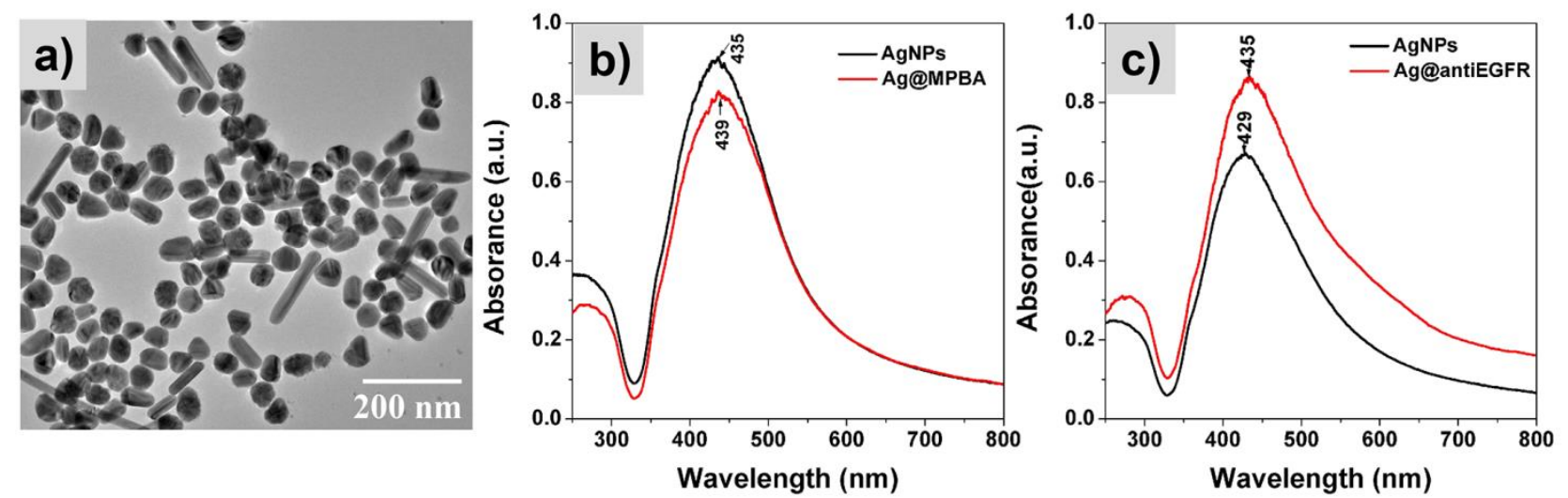

Figure S1. (a) TEM image of AgNPs. (b)-(c) UV-Vis absorption spectra of AgNPs and Ag@MPBA nanoprobe, AgNPs and Ag@antiEGFR nanoprobe, respectively. 


\section{Electromagnetic field distribution of AgNP under FWM}

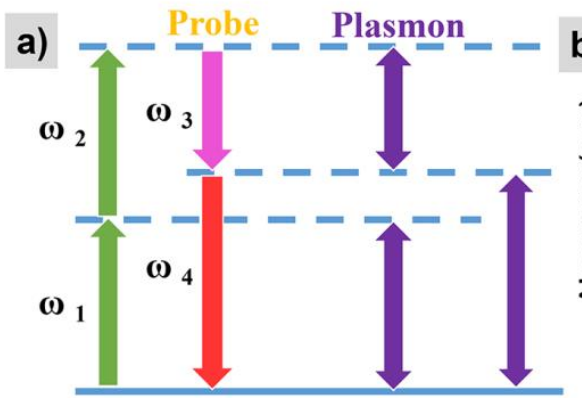

Pump Signal
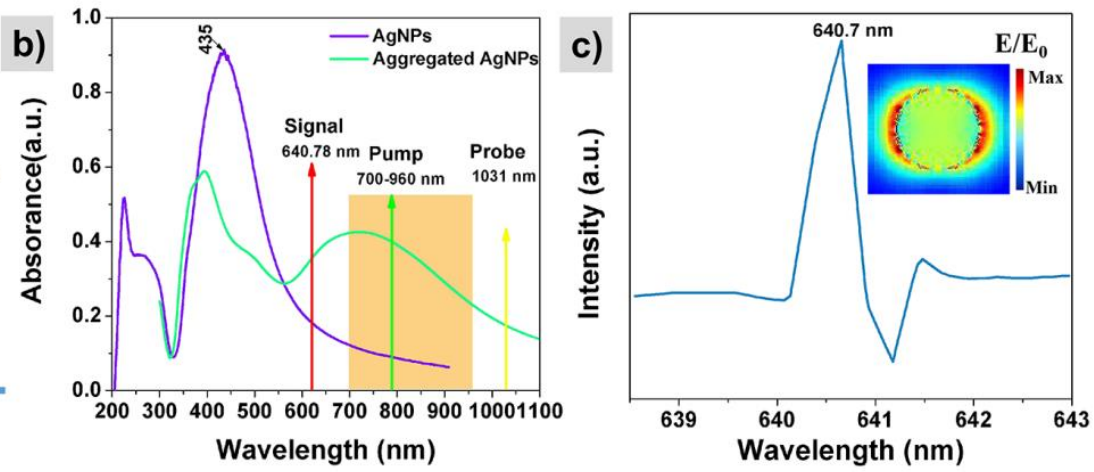

Figure S2. (a). Schematic diagram of plasmon-enhanced four-wave-mixing (PE-FWM) effect. (b). Extinction spectra of the Ag colloid and the AgNPs assembled on a quartz slide. The pink and green lines point out the wavelengths of the probe laser and pump laser, while the red line shows the signal wavelength. (c). The FWM signal of AgNP simulated by FDTD Solution (Lumerical Solutions, Inc.). Electromagnetic field distribution of the plane along the center of a spherical Ag particle with a diameter of $50 \mathrm{~nm}$, simulated by FDTD Solution.

Figure S2a) shows the energy level matching in the plasmon-enhanced four-wave-mixing (PE-FWM) effect. In the present study, owing to the setups that we have, two of $790 \mathrm{~nm}$ lights are set as the pump light and one 1031 $\mathrm{nm}$ is set as the probed light. That is, $\omega_{4}=2 \omega_{1}-\omega_{3}, \omega_{1}=379.48 \mathrm{THz}, \omega_{3}=290.78 \mathrm{THz}$, so $\omega_{4}=468.18 \mathrm{THz}=640.78$ nm.

We prepared an AgNP-assembled film to display how the plasmon resonance effect of Ag work for the FWM effect. The negatively charged AgNPs were assembled on the surface of the hydroxylated glass slide based on the electrostatic interactions provided by the positively charged PDDA. The UV-Vis absorption spectra of Figure S2b show that the assembled AgNPs have a wide band in the range of $600-900 \mathrm{~nm}$, which is different from the plasmonic feature of the Ag colloid that only has one plasmon band at $435 \mathrm{~nm}$. This broadband is caused by the aggregation state of AgNPs on the slide, which is a similar form of AgNPs on the cell surface. The new absorption peaks between 600-900 nanometers precisely resonate with the wavelengths of the pump and probe lasers and the FWM signal, which provides the possibility for improvement of FWM signals based on the LSPR effect (Figure S2a).

The emission of the FWM signal was simulated by the 3D finite-difference time-domain (FDTD) numerical method via a commercial software (Lumerical Solutions, Inc.). The incident lights are three TFSF Source with two 
frequency of $379.48 \mathrm{THz}(790 \mathrm{~nm})$ and a frequency of $290.78 \mathrm{THz}(1031 \mathrm{~nm})$. All the incident lights are set by time domain with the pulse length of $2000 \mathrm{fs}$, offset of $4000 \mathrm{fs}$ and amplitude of $5.0 \times 10^{8}$. In the simulations, the mesh size is chosen to be $1 \mathrm{~nm}$ along the $\mathrm{x}^{-}, \mathrm{y}-$, and $\mathrm{z}$-directions. For the single-particle simulation, the PML boundary condition is used along with all directions. Luminous intensity and electromagnetic field distribution are collected by 2D DFTM power monitor and DFTM monitor, respectively. Since there is no light source at the wavelength monitored by the monitor, to reduce the noise, the spectrum is exported through scripts rather than software. The third-order nonlinear susceptibility $\chi(3)$ of $\mathrm{Ag}$ is taken from G. S. Boltaev's work, which is $2.6 \times 10^{-9}$ esu. ${ }^{[4]}$ The simulated FWM signal is achieved at the wavelength of $640.7 \mathrm{~nm}$ (Figure S2c), which is consistent with the calculated wavelength as we stated above. The electromagnetic field of a spherical Ag particle with a diameter of $50 \mathrm{~nm}$ is presented in Figure S2c). It can be observed the strong electric field is located on the side surface of the Ag sphere, allowing a large gain for the FWM signal. 


\section{Single-cell encapsulation in droplets}

Three different types of cells (HepG2, HeLa, LO2) were incubated in an incubator with $5 \% \mathrm{CO}_{2}$ at $37{ }^{\circ} \mathrm{C}$ for $48 \mathrm{~h}$. Then, the DMEM medium was replaced by two mixture of Iscove's Modified Dulbecco's Medium (IMDM, Gibcos): Ag@MPBA (v\%: v\%)= 9:1 and IMDM: Ag@anti-EGFR (v\%: v\%) =9:1, respectively. Two mixture was incubated with these cells for $1 \mathrm{~h}$, respectively. Then, the samples were washed by phosphate-buffered saline (PBS) for 3 times to remove unbound Ag@MPBA and Ag@anti-EGFR, respectively. Cells were digested with tryptase (wt $0.25 \%$ ) for $2 \mathrm{~min}$. To further remove the unattached probes on the cell surface, the cells were centrifuged at $1500 \mathrm{rpm}$ for $3 \mathrm{~min}$. Then, to prepare the cell suspension, cells were re-suspended in glucose-free medium (IMEM): Optiprep medium $=5.25: 1(\mathrm{v} \%: \mathrm{v} \%)$ mixture. In our experiment, encapsulation rate with a single cell is roughly $21 \%$ by controlling the cell density at $3.0 \times 10^{6}$ cells per mL (Fig. S3), which agrees on David A. Weitz's study in which the achievement for a single cell in one droplet follows the Poisson statistics. ${ }^{[5]}$

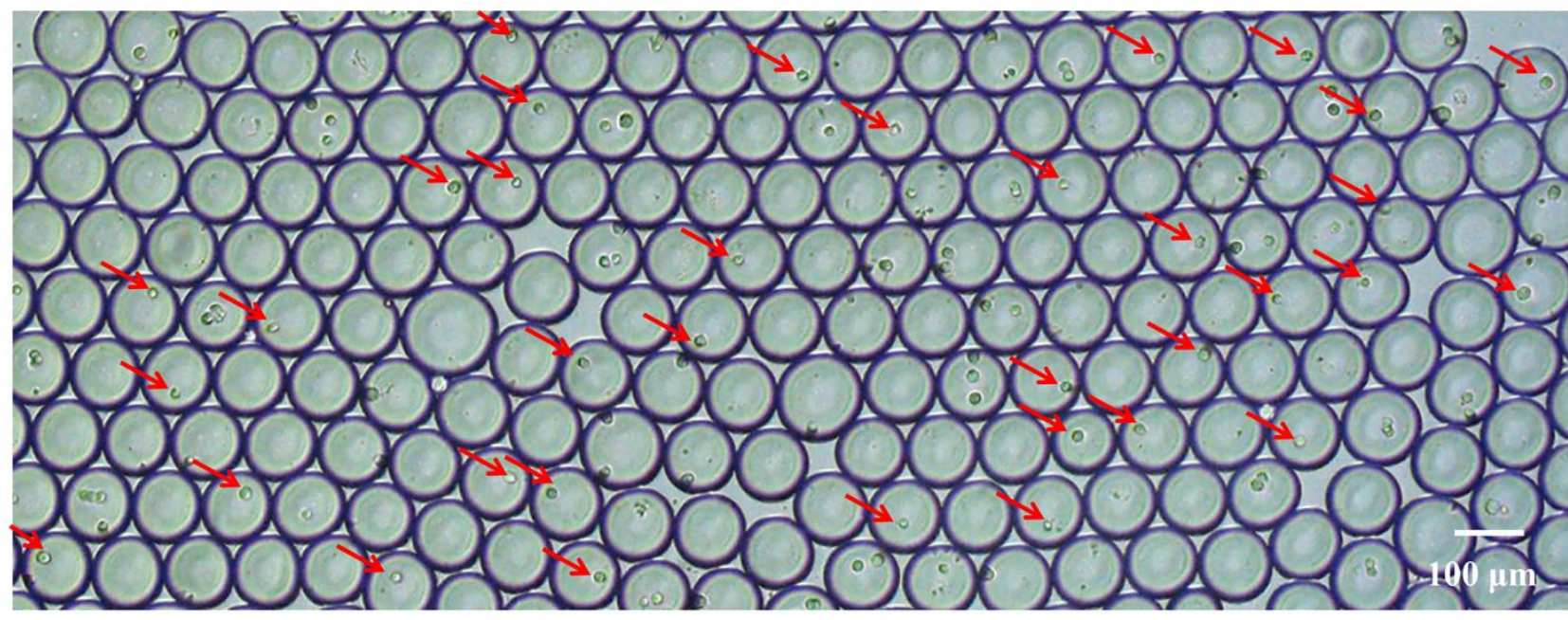

Figure S3. Image of droplets generated with a T-shaped microfluidic chip. Scale bar represents $100 \mu \mathrm{m}$. Red arrows highlight the droplets with one cell. The droplet size was controlled to be about $100 \mu \mathrm{m}$. The flow rate of the carrier-oil phase was $1050 \mu \mathrm{L} / \mathrm{h}$ and the cell suspension phase was $58 \mu \mathrm{L} / \mathrm{h}$.
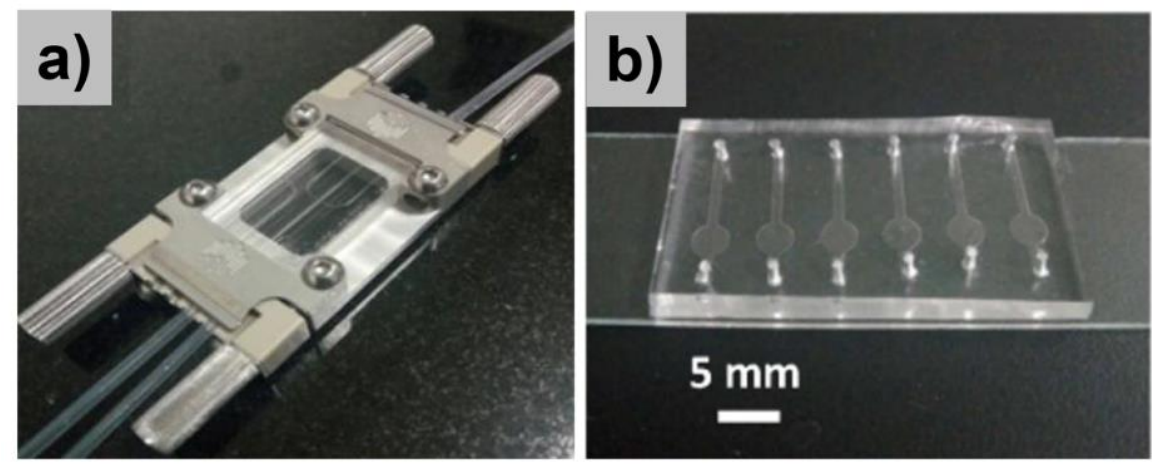

Figure S4. (a). A T-shaped glass chip was used to generate microdroplets. (b). A self-made PDMS-glass chip with 
six array chambers was used for collecting droplet arrays for imaging.

A T-shaped glass chip (Anglian Business Park, Royston, UK) was used to generate microdroplets in this study (Figure S4 a). Fluorinert HFE-7500 fluorocarbon oil is used to suspend droplets. To stabilize the droplet, a concentration of $2.0 \%(\mathrm{w} / \mathrm{w})$ perfluoropolyether-polyethylene glycol (PFPE-PEG) block copolymer surfactant was added to the suspended oil. ${ }^{[6]}$ For drop formation, the syringe pump controller (Baoding Lange TS-2A type) was used to control the syringe pump (LSP01-1A type) to make the flow rate of the carrier-oil phase was $1050 \mu \mathrm{L} / \mathrm{h}$ and cell suspension phase was $58 \mu \mathrm{L} / \mathrm{h}$. Ultimately, the water-in-oil type microdroplets were prepared and the droplet size was controlled to be about $100 \mu \mathrm{m}$. Simultaneously, the droplets were observed under a microscope and collected in a glass vial, and we injected the collected droplets into a self-made PDMS-glass chip with six array chambers (Figure S4 b) to form a droplet array for dark imaging and PE-FWM imaging. The details for the PDMS-glass chip has been stated in our previous publication. ${ }^{[2]}$ 


\section{Dark-field imaging and bright-field imaging (particle-finder)}

The dark-field images of the Ag@MPBA binding with SA on the LO2 cell surface were collected by the self-built imaging platform in our group. ${ }^{[7]}$

The Ag@MPBA was incubated with HeLa cells for one hour and then fixed for bright-field imaging. The bright-field image processing with "Particle Finder" of LabSpec6 software (HORIBA, France). Specifically, we sent the bright-field image to the "Particle Finder" of LabSpec6 software. The number of adsorbed nanoparticles on the cell surface was calculated automatically by adjusting the threshold. 


\section{Fluorescence imaging of EGFR on cell surface}

HepG2 cells were cultured on the coverslips in DMEM with $10 \%$ fetal bovine serum (FBS). They were incubated in an incubator with $5 \% \mathrm{CO}_{2}$ at $37{ }^{\circ} \mathrm{C}$ for $24 \mathrm{~h}$. The HepG2 cells on the coverslips were washed with blocking solution (PBS with 5\% FBS) for three times, and fixed in 4\% formaldehyde solution for 20 min. All coverslips were washed with blocking solution three times and then were added $1.0 \mathrm{~mL}$ of blocking solution for $2 \mathrm{~h}$ to avoid non-specific adsorption. Then, the EGFR Monoclonal Antibodies (111.6) (anti-EGFR, $5 \mu \mathrm{g} / \mathrm{mL}$ ) were added to the coverslips and incubated with the HepG2 cells in the incubator for $1 \mathrm{~h}$. After that, we washed all coverslips five times with a blocking solution to get rid of unbound anti-EGFR. Next, the Alexa Fluor® 488 donkey anti-mouse $\operatorname{IgG}(\mathrm{H}+\mathrm{L})$ antibody $(2 \mu \mathrm{g} / \mathrm{mL})$ was added to the coverslips and incubated with the HepG2 cells in the incubator for $1 \mathrm{~h}$. Then, Hoechst 33342 (dilution ratio 1:1000) was added and shaken for 10 min to stain the nucleus. Finally, the cells were sealed for fluorescent imaging. The Alexa Fluor ${ }^{\circledR} 488$ donkey anti-mouse $\operatorname{IgG}$ $(\mathrm{H}+\mathrm{L})$ antibody was excited by a $488 \mathrm{~nm}$ laser, and Hoechst 33342 was under $405 \mathrm{~nm}$ excitation. 


\section{FWM and TPL phenomena from AgNPs}

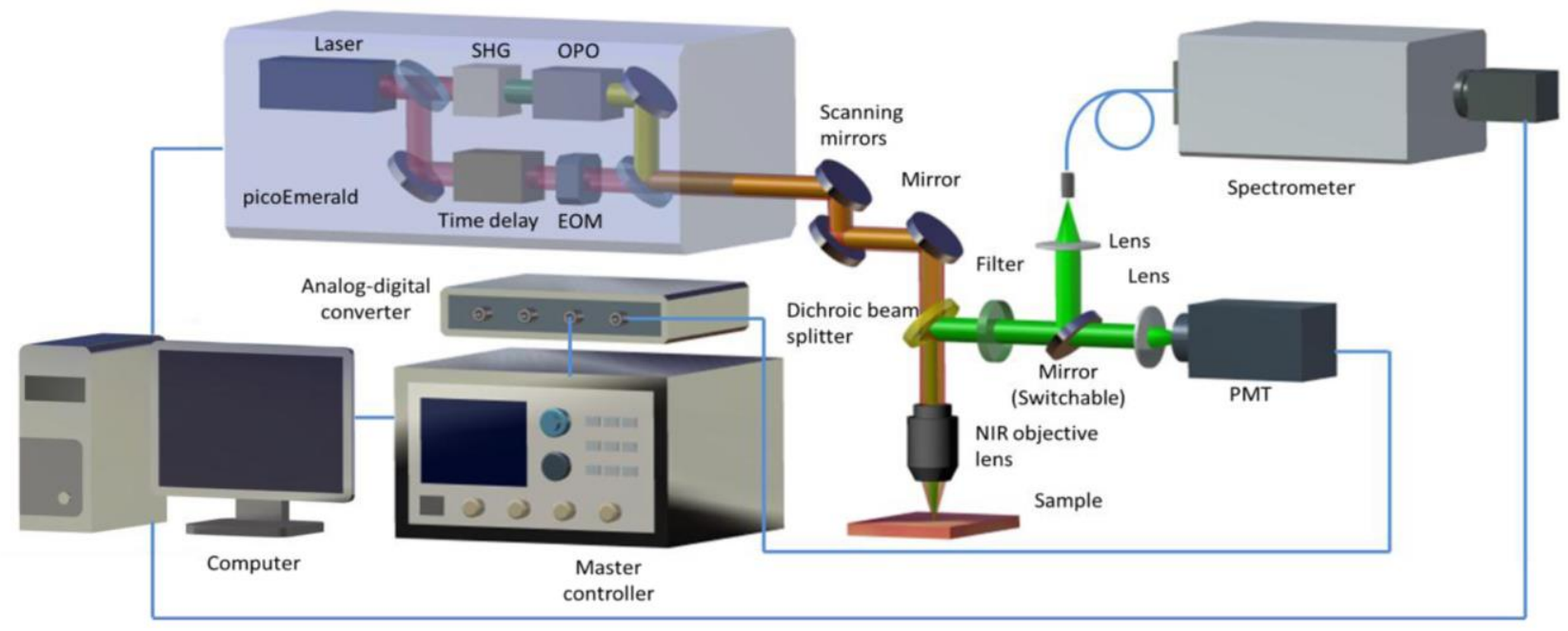

Figure S5. The hardware schematic diagram of the FWM and TPL detection system.

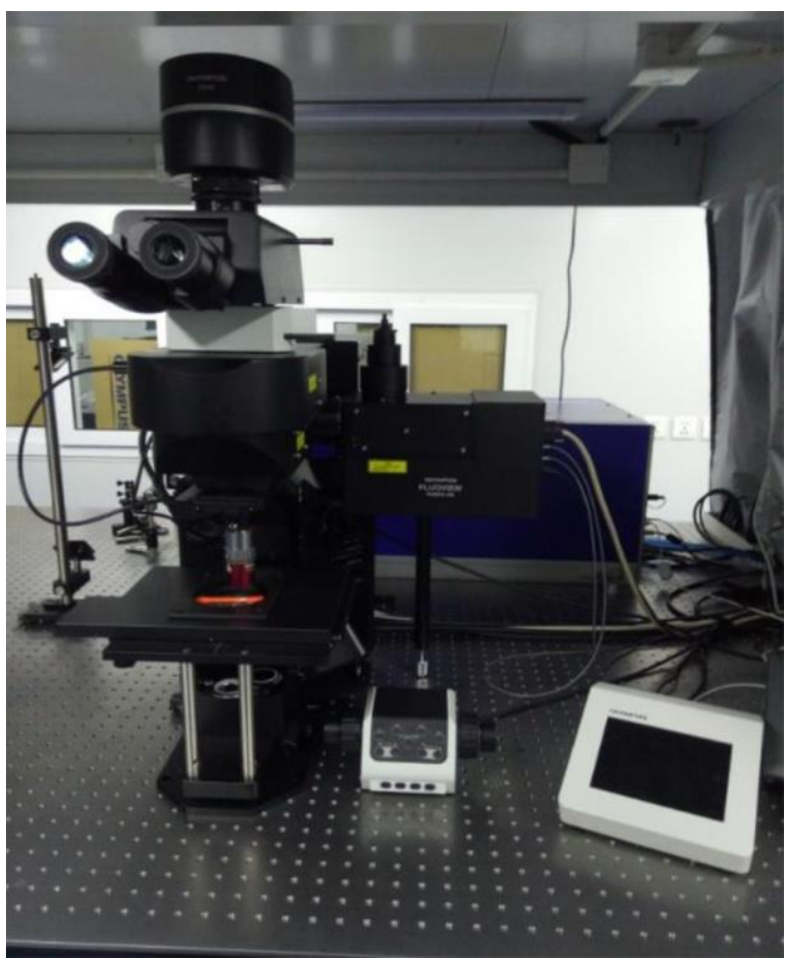

Figure S6. A photo of the multiphoton microscope for the FWM and TPL spectroscopic measurements.

The instrument for FWM imaging is a self-built setup, which is composed by a picosecond laser (picoEmerald, APE) with double beam output ability and a multi-photonic microscope (FVMPE-RS, Olympus). Two lasers with different wavelengths $(1031 \mathrm{~nm}$ and 700-960 nm) enter the microscope in a coaxial form and focus on the sample through the objective lens. The four-wave mixing signal produced by the sample is collected by the objective lens 
and its intensity is measured by the photomultiplier (PMT).

In order to detect FWM and TPL spectra of AgNPs, we modified the commercial multiphoton microscope (FVMPE-RS, Olympus) by fixing a spectrometer (USB4000, Ocean optics, USA). Figure S5 shows its optical configuration and working principle. When detecting the FWM signal, the picosecond laser (picoEmerald, APE, Germany) generates two laser beams. In order to achieve coherence when two laser beams arrive at the sample, an optical time delayer is installed in the laser $(790 \& 1031 \mathrm{~nm})$. The two laser beams are coaxial and output from the laser to a scanning mirror. Then, the laser beams are reflected by a mirror and changed to the downward direction. After going through a $45^{\circ}$ dichroic beam splitter (690 nm, long-pass), the laser is focused on the sample by a near-infrared objective lens (20x, Mitutoyo). The FWM signal generated by the sample is collected and collimated by the same objective lens. After going through a short pass filter, the FWM signal is reflected upward by a switchable mirror, coupled to an optical fiber, and finally arrives in a spectrometer (USB4000, Ocean optics, USA).

Compared with the detection of FWM signal, TPL signal only uses one laser $(790 \mathrm{~nm})$ for measurements. In addition, the laser is focused on the sample through a $50 \times$ objective lens (Olympus), and other settings are consistent with the FWM signal detection.

The FWM and TPL spectra of an AgNP-assembled film were recorded. We used a 20x objective for the TPL signal collection, and almost no signal was generated. When we switched to a 50× objective, the AgNPs became observable (Figure S7b). Its spectral profile is generally broad. The FWM signal collected with a 20x objective shows remarkably strong and gives a narrow spectral width (Figure S7a). The peak position is precisely corresponding to the coherent frequency. It should be noted that all our studies and experiments collected FWM signals under a 20x objective. So, the spectra in Figure S7 prove that the signal given by AgNPs is not originated from TPL, but FWM.
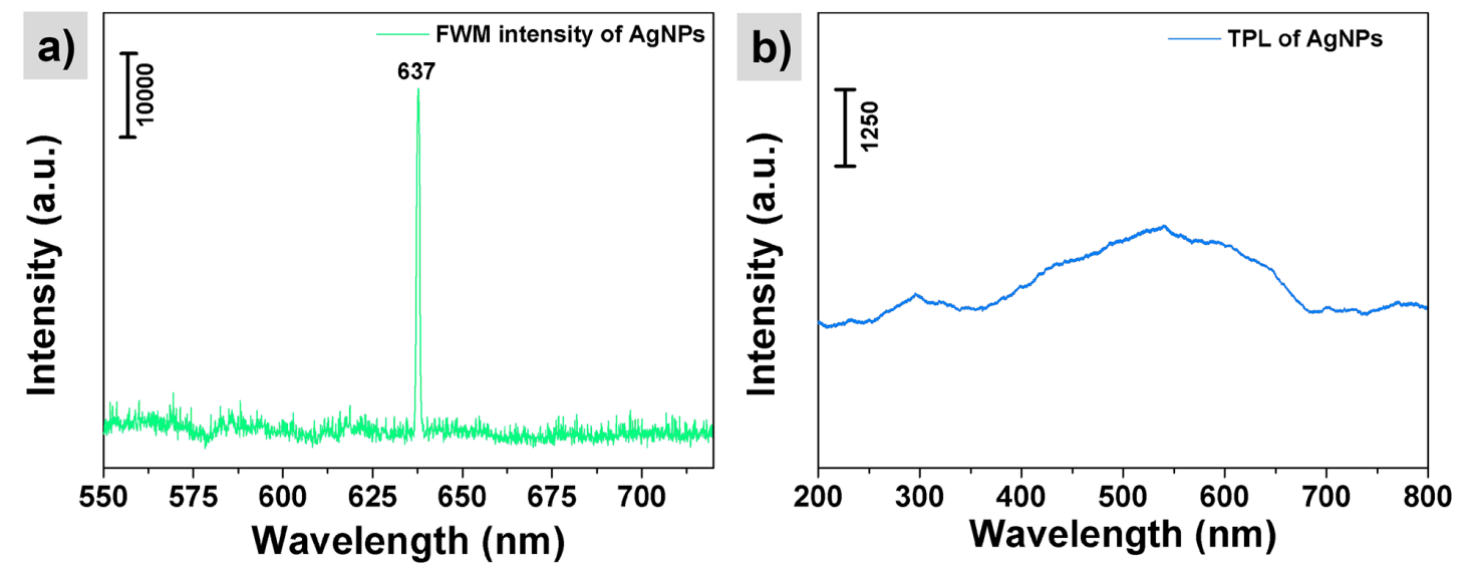

Figure S7. The FWM intensity (a) and the TPL intensity (b) of AgNPs. 


\section{FWM imaging of single cell contained droplet array and automatic image processing by Image J}

To avoid the non-specific adsorption of our designed nanoprobes, AgNPs without any surface modification were used instead of the Ag@MPBA for cell culture. The FWM image of cells in droplets (Figure S9) shows there are no signals from AgNPs, indicating the specificity of nanoprobes toward SA and the non-specific adsorption can be avoided.

To take the FWM images, the scanning mirror arranged in the microscope can realize the laser spot scanning on the surface of the sample point by point. The computer records the FWM signal intensity of each point to rebuild images.

Image $\mathbf{J}$ software was used to automatically calculate the integrated density of cells in each droplet. Briefly, the FWM image was converted to the image type of 8-bit (Scheme 1b), and the "Invert" was selected in the Edit. Next, the "Threshold" window was selected from the "Adjust" window under the Image. By adjusting the threshold through the "Threshold" window, we set the threshold at 0-237, and cells whose brightness exceeds the threshold will be counted. Then, the "Analyze particles" was clicked under the Analyze window, we set the size of cancer cell lines is 100-Infinity.and normal cell line is 50-Infinity. Finally, the integrated density of adsorbed nanoparticles on the surface of all cells in each image was automatically calculated. Subsequently, the Integrated density values (IntDen) of 202 groups for three cell lines were respectively treated with a T-test.

T-test was used to analyze differences in SA and EGFR distribution between cancer cells and normal cells. *** means significantly different at the $\mathrm{p}$-value $<0.001$. ** means significantly different at the $\mathrm{p}$-value $<0.01 . *$ means significantly different at the p-value $<0.05$, and ns means the differences are not significant. 


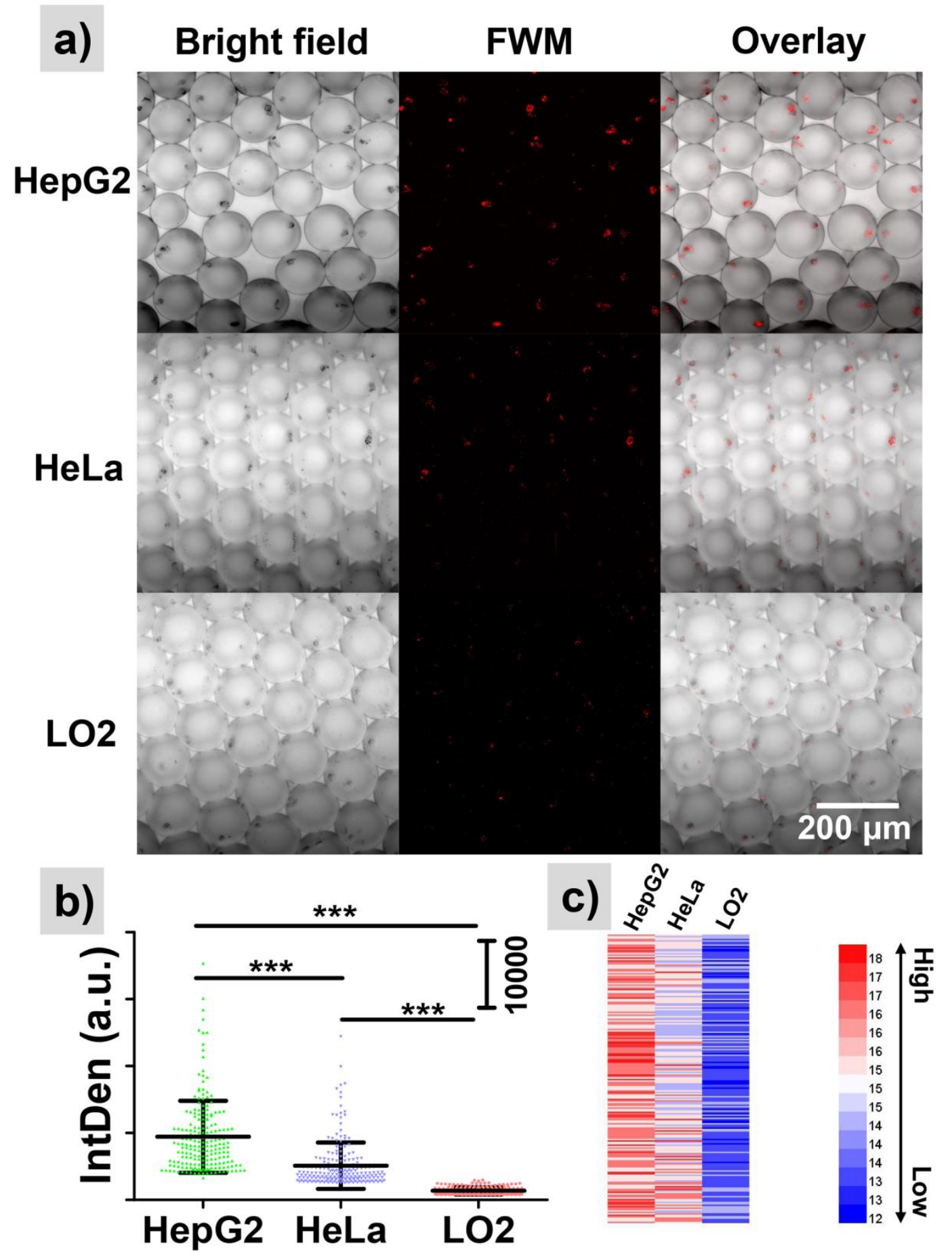

Figure S8. (a) The bright-field, FWM and overlay images of the HepG2, HeLa and LO2 cells with the Ag@MPBA nanoprobes in the microdroplet arrays, respectively. (b) T-test analysis of the IntDen of SA from each cell line. 202 groups of cells on each cell line processed with Image J software are plotted. The error bars represent the standard deviations of mean IntDen. ${ }^{* * *}$ means significantly different at the p-value $<0.001$. (c) Heatmap of SA from three cell lines. Each stripe represents a cell. 


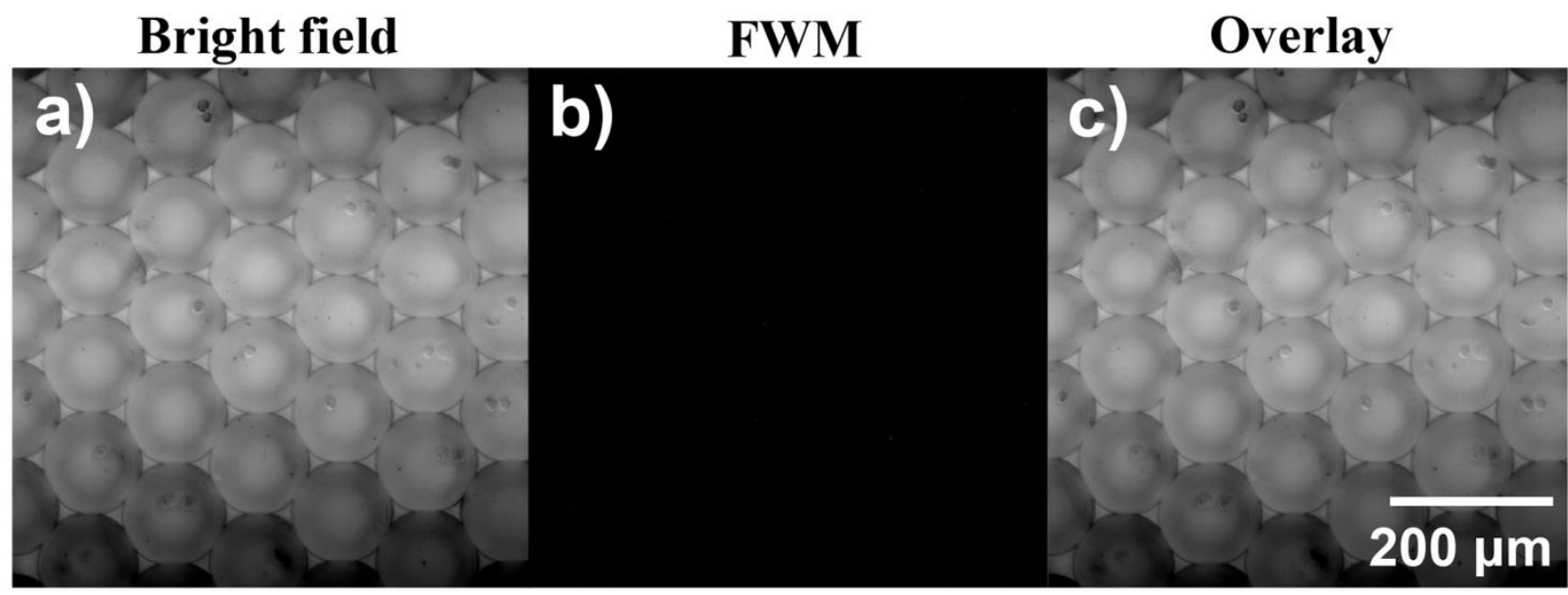

Figure S9. (a)-(c) The bright-field, FWM and overlay images of LO2 cells in the microdroplet array after they were incubated with AgNPs for $1 \mathrm{~h}$ in the microdroplet array. 


\section{References}

[1] Lee, P. C.; Melsel, D. Adsorption and Surface-Enhanced Raman of Dyes on Silver and Gold Sols. J. Phys. Chem. 1982, 86, 3391-3395.

[2] Cong, L. L.; Liang, L. J. et al. Distinguishing cancer cell lines at a single living cell level via detection of sialic acid by dual-channel plasmonic imaging and by using a SERS-microfluidic droplet platform. Microchimica Acta. 2019, 186, 367-376.

[3] Kubota, T.; Kuroda, S. et al. Nanomedicine: Nanotechnology, Biology, and Medicine, 2018, 14, 1919-1929.

[4] Boltaev, G. S.; Ganeev, R. A.; et al. Strong Third-Order Optical Nonlinearities of Ag Nanoparticles Synthesized by Laser Ablation of Bulk Silver in Water and Air. Appl. Phys. A. 2018, 124 (11), 766-780.

[5] Koster, S.; Angile, F. E. et al. Drop-based microfluidic devices for encapsulation of single cells. Lab Chip. 2008, $8,1110-1115$.

[6] Holtze, C.; Rowat, A. C. et al. Biocompatible surfactants for water-in-fluorocarbon emulsions. Lab Chip. 2008, $8,1632-1639$.

[7] Li, H. B.; Wang, H. L. et al. Note: Raman microspectroscopy integrated with fluorescence and dark field imaging. Rev. Sci. Instrum. 2014, 85, 056109. 